Long-Term Disaster Resilience: A Research Gap

Dr. Caroline Spencer, Dr. Saadia Majeed, Mr. Dudley McArdle, Dr. Deb Parkinson, Professor Frank Archer

Monash University, Clayton, Australia

Introduction: This research identified a gap in understanding the lived experience of long-term disaster resilience (LTDR). Increasing disasters could influence more people. Therefore, understanding LTDR becomes imperative. Little research documents men and women's reflections following disasters. Current research highlights survivors' mental health, particularly clinical diagnoses like PTSD. Research remains limited on the social impacts long after disasters.

Aim: Research aimed to identify a gendered perspective of the lived experience about what contributes to LTDR three years after Ash Wednesday in 1983, the Victorian floods in 1993 and 2010-11, and the 2009 Black Saturday fires.

Methods: A comprehensive, systematized search was conducted of peer-reviewed, grey, and secondary literature for a narrative review and thematic analysis.

Results: 106 references were identified. After removing duplicates and papers not fitting the inclusion criteria, two papers met the criteria. However, two borderline papers were included due to the closeness of the timeframe and brevity of research available.

Discussion: Most research is related to the immediate aftermath or short-term resilience. Papers provided no specific attributes to enhance the lived experience of LTDR as it related to gender. However, factors that could enhance the lived experience of LTDR were drawn from six themes in sociological studies. Presumptive interpretations were made about what factors may provide insight into the social and contextual issues of LTDR. The literature dearth identified the need for longterm disaster resilience research. The most striking conclusion drawn from themes tells how people perceived the way a disaster and the ensuing period affected their personal relationships and circumstances. Overall, positive experiences strengthened their resilience while negative experiences hindered their resilience. While the review resulted in a disappointing outcome, the dearth of LTDR research lacked any reference to gender but confirmed research opportunities for innovative research that could influence policy and practice.

Prehosp. Disaster Med. 2019;34(Suppl. 1):s21

doi:10.1017/S1049023X19000608

\section{Overcoming Ambiguity: Conflict Between Emergency Warning Messages and Socio-Environmental Cues} Dr. Paula Dootson 1,2, Associate Professor Dominique Greer ${ }^{1,2}$, Ms. Sophie Miller ${ }^{1,2}$, Professor Vivienne Tippett ${ }^{1,2}$

1. QUT, Brisbane, Australia

2. Bushfire and Natural Hazards CRC, Melbourne, Australia

Introduction: Emergency services are not the only source of information that the public uses when considering taking action during an event. There are also environmental cues, information from the media, or actions by peers that can influence perceptions and actions. When cues from different information sources are in conflict, it can cause uncertainty about the right protective action to take.

Aim: Our research responds to concerns that conflicting cues exacerbate community non-compliance with emergency warnings. Methods: The sample consisted of 2,649 participants who completed one of 32 surveys.

Results: The findings from this project confirmed emergency services agencies' suspicions that conflicting cues can affect information processing and risk perceptions, and therefore prevent people from taking appropriate protective action. The results were reasonably consistent across fire and flood scenarios, suggesting the problem of conflicting cues is not hazardspecific. When presented with consistent cues, participants were more likely to evacuate, perceive risk about the event, share information with friends, family, and peers, find emergency warnings to be effective, and comprehend information. When faced with conflicting cues, participants were more likely to seek out additional information. It affected their information processing and self-efficacy. The results did not change for people of different ages, native language, country of birth, or posthazard experience. This is contrary to most emergency literature research findings, which show that individual differences play a role in impacting propensity to take protective action. However, there does appear to be a significant gender effect. These results require further exploration.

Discussion: These findings may be used to assist emergency services agencies to tailor community warnings during timecritical situations, and develop ways to mitigate ambiguity caused by conflicting cues to encourage protective action in order to save lives and properties.

Prehosp. Disaster Med. 2019;34(Suppl. 1):s21

doi:10.1017/S1049023X1900061X

\section{Practice, Experience, and Prospect of Disaster Medicine in China}

Dr. Shike Hou, Dr. Haojun Fan, Dr. Yanmei Zhao

Tianjin University, Tianjin, China

Introduction: In recent years, the development of disaster medicine has made rapid progress in China after the Wenchuan earthquake in 2008. China formed a more systematic and specialized health emergency force. In addition, the Ministry of Emergency Management was established In 2018, which demonstrated that the Chinese government is paying more attention to the disaster rescue work. In this report, the practice, experience, and prospect of disaster medicine in China was reviewed. Aim: To explore the current status and prospects of disaster medicine development in China.

Methods: The literature research method was used to analyze literature at home and abroad. It was used to retrospectively analyze the rescue experience of Chinese medical rescue teams, sort out the current situation of disaster medical disciplines and rescue teams, and propose disaster medical development recommendations suitable for China's national conditions.

Results: After years of construction, China has formed a more systematic and specialized health emergency force, but the personnel, equipment, plans, and training of the professional 
rescue team need to be improved. The discipline system of disaster medicine in China is still lagging behind, and it is still unable to meet the needs of the rescue situation at home and abroad.

Discussion: Disaster medicine is the area of medical specialization serving the dual areas of providing health care to disaster survivors and providing medically related disaster preparation, disaster planning, disaster response, and disaster recovery leadership throughout the disaster life cycle. It requires multidisciplinary intervention, integration, and application. A forwardlooking perspective must be strengthened on the discipline and team building of disaster medicine.

Prehosp. Disaster Med. 2019;34(Suppl. 1):s21-s22

doi:10.1017/S1049023X19000621

\section{Reviewing Disasters: Hospital Evacuations in the United States from 2000 to 2017}

Miss. Aishwarya Sharma ${ }^{1}$, Dr. Sharon Mace ${ }^{2}$

1. Ohio University Heritage College of Osteopathic Medicine, Warrensville Heights, United States

2. Cleveland Clinic Lerner College of Medicine at Case Western Reserve University, Cleveland, United States

Introduction: Between 2000 to 2017, there were over 150 hospital evacuations in the United States. Data received from approximately 35 states were primarily concentrated in California, Florida, and Texas. This analysis will provide disaster planners and administrators statistics on hazards that cause disruptions to hospital facilities.

Aim: The aim of this study is to investigate US hospital evacuations by compiling the data into external, internal, and manmade disasters thus creating a risk assessment for disaster planning. Methods: Hospital reports were retrieved from LexisNexis, Google, and PubMed databases and categorized according to evacuees, duration, location, and type. These incidents were grouped into three classifications: external, internal, and manmade. Both partial and full evacuations were included in the study design.

Results: There were a total of 154 reported evacuations in the United States. 110 (71\%) were due to external threats, followed by $24(16 \%)$ man-made threats, and $20(13 \%)$ internal threats. Assessing the external causes, 60 (55\%) were attributed to hurricanes, $21(19 \%)$ to wildfires, and $8(7 \%)$ to storms. From the internal threats, $8(40 \%)$ were attributed to hospital fires and 4 (20\%) chemical fumes. From the man-made threats, $6(40 \%)$ were attributed to bomb threats and $4(27 \%)$ gunmen. From the 20 total reported durations of evacuations, 9 (45\%) lasted between 2 to 11:59 hours, 6 (30\%) lasted over 24 hours, and $5(25 \%)$ lasted up to $1: 59$ hours.

Discussion: Over 70\% of hospital evacuations in the US were due to natural disasters. Compared to 1971-1999, there was an increase in internal and man-made threats. Exact statistics on evacuees, durations, injuries, and mortality rates were unascertainable due to a lack of reporting. It is critical to implement a national registry to report specifics on incidences of evacuations to further assist with disaster and infrastructure planning.

Prehosp. Disaster Med. 2019;34(Suppl. 1):s22

doi:10.1017/S1049023X19000633

\section{"We Just Want to Help" - Nonprofits Contributions to Community Resilience in the Disaster Space \\ Ms. Fiona Roberts, Emeritus Professor Frank Archer, \\ Dr. Caroline Spencer \\ MUARC, Monash University, Clayton, Australia}

Introduction: The National Strategy for Disaster Resilience (NSDR) characterizes resilient communities as having strong disaster and financial mitigation strategies, strong social capacity, networks, and self-reliance. Nonprofit organizations (NPOs) embrace many characteristics of a disaster resilient community. NPOs do not operate for the profit of individual members. Community groups like Lions and Rotary Club have long histories, and while not established to respond to disasters, they frequently have heavy involvement in preparing for or recovering from, disasters.

Aim: The study aims to address the question, "What is the potential role of nonprofit organizations in building community resilience to disasters?"

Methods: An applied research project was carried out, using theories of resilience, social capital, and the Sendai framework to conceptualize the frameworks and guide the process. Qualitative research methods, thematic analysis, and case studies helped identify Lions, Rotary, and Neighbourhood Houses Victoria strengths, barriers, and enablers.

Results: Research demonstrated how NPOs made significant contributions to building communities' resilience to disasters. NPOs facilitate three Sendai guiding principles of engaging, empowering, and enabling the community to build disaster resilience. Actions included raising awareness to disaster risk, reducing disaster risk, helping prepare for disasters, and contributing to long term disaster recovery. NPO strengths included local knowledge, community trust, and connections, which matched characteristics listed in the NSDR for a disaster resilient community. However, barriers to participation included traditional emergency services ignoring NPOs, lack of role definition, and lack of perceived legitimacy.

Discussion: As the first Australia research to scientifically analyze the contributions of these NPOs to build community resilience, before, during and after disaster, this study enhances understanding and recognition of NPOs and assists in identifying means to facilitate their disaster resilience activities and place them more effectively within Emergency Management strategic processes. Greater utilization of such assets could lead to better community outcomes.

Prehosp. Disaster Med. 2019;34(Suppl. 1):s22

doi:10.1017/S1049023X19000645 